\title{
IS HIGH-FREQUENCY STIFFNESS A MEASURE FOR THE NUMBER OF ATTACHED CROSS-BRIDGES ?
}

\author{
B.J.J.J. van der Linden', H.F.J.M. Koopman' ${ }^{\text {, P.A. Huijing }}{ }^{1.2}$ and H.J. Grootenboer ${ }^{1}$ \\ 'Biomedical Institute of Technology, University of Twente, P.O. Box 217.7500 AE. Enschede. The \\ Netherlands; e-mail: b.j.j.j.vanderlinden@wb.utwente.nl \\ ${ }^{2}$ Faculteit Bewegingswetenschappen. Vrije Universiteit. Amsterdam. The Netherlands.
}

\begin{abstract}
Muscle stifness is an important property for movement cumbol. Stiffiness is a measure for the resistance against mechanical disturbances in muscular-skeletal sysicms. In erencral muscle stiffness is assumed to depend on the number of altached cross-bridges. It is not possible to measure this number in vive or vitro. In experiments, high frequency perlurbations are used 10 oblain a measurement of stiffiness. In thes paper a simulation study is presented concerning the correlation between the number of attached cross-bridges and high-frequency stiffness. A model based on the slidingfilament theory was used for the simulation of dynamic contractions. It is concluded that these two methods of musch stilthess determination do nor yicld compatible results during knghening.
\end{abstract}

\section{Introduction}

Muscle stillness is a measure for stability against possible perturbations during movement. Theretore, many efforts are made to determine the stiffness of muscle and fibers (e.g. 111 . 121). In experiments the so called high-frequency stiffness is measured. which is based on the assumption that the number of atached cross-bridges is not affected by small changes in length. The elasticity of the altached cross-hridges and series clastic structures determine the muscle stiffness. Because the woss-bridges act in parallel in a sarcomere. the number of atlached cross-bridges is proportional to stiffness 131 . In this papet we focus on the relation between stiffness based on the number of allached cross-bridges compared to high-irequency silfmess. With a model based on the sliding-filament theory 1) Huxley [4] we determined stiffness in both ways under sumetric and sokmetucondituons.

\section{Methods}

The model is hased on the partial differential equation (PDE) of Huxley [4]. which describes cross-bridge cycling during muscle contraction. This model was extended with a filament overlap function which defines the maximal number of available cross-bridges for attachment. Some further moditications were made to the original Huxley model to account for physiological structures in a sarcomere: a series clastic clement (a linear spring and a dashpot to acount for the minor viscous effects. were incorporated.

The stiffness measurement based on the number of crossbridges is just a simple count of the attached cross-bridges which are recorded in the cross-bridges distribution. The high frequency stilfness can be calculated from the force changes during a sinusoidal tength perturbation of the sarcomere.

The contraction protocol was divided into thee phases. The lirst phase is an isometric phase during which the tetanic force is developed. The second phase is characterized as the isokinetic phase. this phase is absent during the isometric contractions. During the final phase (e.g. post-stretch) the active isonctric force is allowed to redevelop.

\section{Results}

The results of the isometric and concentric contractions are consistent with what can be expected. The high-frequency stiffiness is proportional to the number of attached crossbridges. During the simulation of the eccentric contraction we do not see this proportionality. The model does predict a force enhancement during lengthening. The enhancement is comparable to tindings of Sugi and Tsuchiya [2]. But. during the lengthening phase a distinct decrease of the number of attached cross-hridges can be observed. During the active 
pust-stretch phase the number of atached cross-bridges recovers to a number equivalent to the new isometric level. When the same cuntraction protocol with length perturbations is used. a corresponding number of attached cross-bridges during the whole contraction can be observed. In contrast with this result, a considerable increase in the high-frequency siffiness is calculated during lengthening. During the active post-stretch phase the high-frequency stiflness reduces to an isometric level again.

\section{Discussion}

The simulations show a discrepancy between stifiness of a sarcomere and the predicted number of attached cross-bridges during lengthening. This is consistent with findings of Cholewicki and MeGill [5]. Their simulations demonstrated wal the number of attached cross-bridges decreased during isokinetic contractions. The high-frequency stiffness cialculated with the model shows a remarkable resemblance with experimental data of Sugi and Tsuchiya [2].

Based on experimental data [2] and our findings it is very likely that high-frequency stiffness is proportional to muscle force. Moreover, the reduced number of attached crossbridges during lengthening indicates an incrcase in exerted force per cross-bridge. Finally. it is concluded that highfrequency stifliness during lengthening is not compatible with stiflness based on the number of altached cross-bridges.

\section{References}

(1) Julian. F.J. and M.R. Sollins (1975) "Variation of muscle stiffness with force at increasing speeds of shortening": J Gen Physiol; 66; 287-302.

12] Sugi. H. and T. Tsuchiya (1988) "Muscle stiffness and force changes"; J Physiol: 407: 215-229.

(3) Gordon. A.M. A.F. Huxley and F.J. Iulian (1966) "The variation in isometric tension with sarcomere length in vertebrate muscle fibers": J Physiol: 184: 170-192.

14) Huxley, A.F. (1957) "Muscle structure and theories of contracion": Prog Biophys Biophys Chem: 7: 255318.

[5] Cholewicki, J. and S.M. McGill (1995) "Relationship between muscle force and stiffness in whole mammalian musele: a simulation study" I Biomech Eng; 117: 339-342. 